\title{
High Resolution X-ray Observations of 3C 58
}

\author{
Patrick Slane \\ Harvard-Smithsonian Center for Astrophysics, 60 Garden Street, \\ Cambridge, MA 02138, USA
}

\begin{abstract}
As the presumed remnant of SN 1181, 3C 58 houses one of the youngest known neutron stars in the Galaxy. The properties of this young pulsar and its associated pulsar wind nebula (PWN) differ considerably from those of the Crab Nebula, and may well offer a more typical example of the endpoint of massive star collapse. High resolution X-ray studies reveal structures in the inner nebula that may be associated with the pulsar wind termination shock, a jet that may be aligned with the rotation axis, and other regions of enhanced emission. Spectral variations in the PWN are consistent with the expected evolution of the postshock flow, and complex loops of emission are seen in the nebula interior. Limits on the neutron star surface temperature fall below standard cooling models, indicating that some more rapid neutrino cooling process is required. The outer regions of $3 \mathrm{C} 58$ show thermal emission with enhanced levels of neon, indicative of shocked ejecta bounding the PWN.
\end{abstract}

\section{Introduction}

3C $58(\mathrm{G} 130.7+3.1)$ is a Crab-like nebula believed to be associated with the historical supernova SN 1181. X-ray observations reveal a compact source at the center, and a power law spectrum whose index steepens with radius, characteristic of particle injection from a central pulsar. 3C 58 appears to be quite different from the Crab Nebula in many respects, however. The pulsar wind nebula (PWN) is larger, and the radio luminosity is ten times lower than that of the Crab. The X-ray luminosity is nearly 2000 times lower, perhaps suggesting that any central pulsar has undergone a significant reduction in its injection power. However, recent Chandra HRC observations (Murray et al. 2002) have revealed PSR J0205+6449, the second most energetic pulsar known in the Galaxy, powering the nebula; the characteristic age is $\tau \equiv P / 2 \dot{P} \approx 5 \times 10^{3} \mathrm{yr}$ which, assuming a true age of $\sim 820 \mathrm{yr}$ based on the historical association, implies an initial spin period of $P_{0} \approx 60 \mathrm{~ms}$ - much longer than that inferred for the Crab pulsar.

The emission from the center-most region of $3 \mathrm{C} 58$ is extended, with elongation in the N-S direction, perpendicular to the long axis of the main nebula. The pulsar resides at the center of this compact core nebula, and a jet-like feature protrudes westward. At the western edge resides a narrow radio wisp discovered by Frail \& Moffett (1993), who suggested that this lies along the pulsar wind termination shock. Using the measured value of $\dot{E}=10^{37.6} \mathrm{ergs} \mathrm{s}^{-1}$ and the pressure determined for the nebula interior, the expected radius of the termina- 


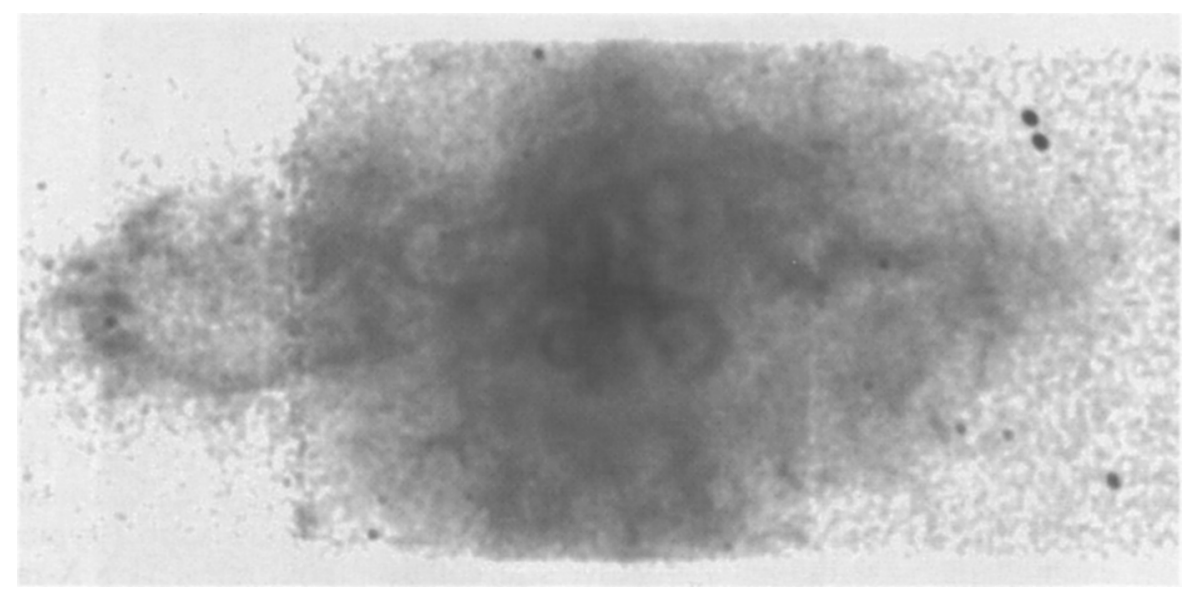

Figure 1. 350 ks X-ray image of 3C 58 taken with the Chandra ACIS detector. The compact region in the center is extended, and reveals a jet-like feature protruding to the west. On larger scales, complex loop structures are observed throughout the PWN.

tion shock is $r_{w} \approx 12^{\prime \prime}$, in good agreement with the north-south extent of the core nebula in 3C 58 (Slane et al. 2002). This suggests a toroidal structure with an inclination angle of $\sim 70^{\circ}$, and with a projected pulsar spin axis in the E-W direction. The elongated shape of the entire PWN is consistent with a pulsar axis oriented in the E-W direction (Begelman \& Li 1992; van der Swaluw 2003).

While unresolved from the compact surrounding nebula, limits on the Xray flux from PSR J0205+6449 provide important constraints on models for neutron star (NS) cooling. The upper limit to the thermal emission from the NS surface falls well below predictions from standard cooling models and appears to require the presence of some rapid cooling mechanism (Slane, Helfand \& Murray 2002). Such rapid cooling may imply an above-average NS mass, for which an increased proton fraction in the interior allows the direct Urca process to proceed (Yakovlev et al. 2002), or may indicate the presence of pion condensates in the NS interior, which also leads to rapid cooling (Tsuruta et al. 2002).

\section{New Observations of $\mathbf{3 C} 58$}

3C 38 was recently observed for 350 ks with the ACIS detector onboard the Chandra X-ray Observatory. The X-ray image, shown in Figure 1 (Slane et al., in preparation) and described in more detail below, reveals a complex structure consisting of loops, elongated features, and broad diffuse emission. The observation was divided into three segments to accommodate interruptions by the radiation zones in the Chandra orbit. An error in on-board commanding resulted in the final segment being carried out with the spacecraft dither turned off for the full $170 \mathrm{ks}$ duration, resulting in no exposure in regions corresponding to gaps between the CCD chips or to bad column regions in the CCDs for this segment; these result in faint vertical features evident in Figure 1. 

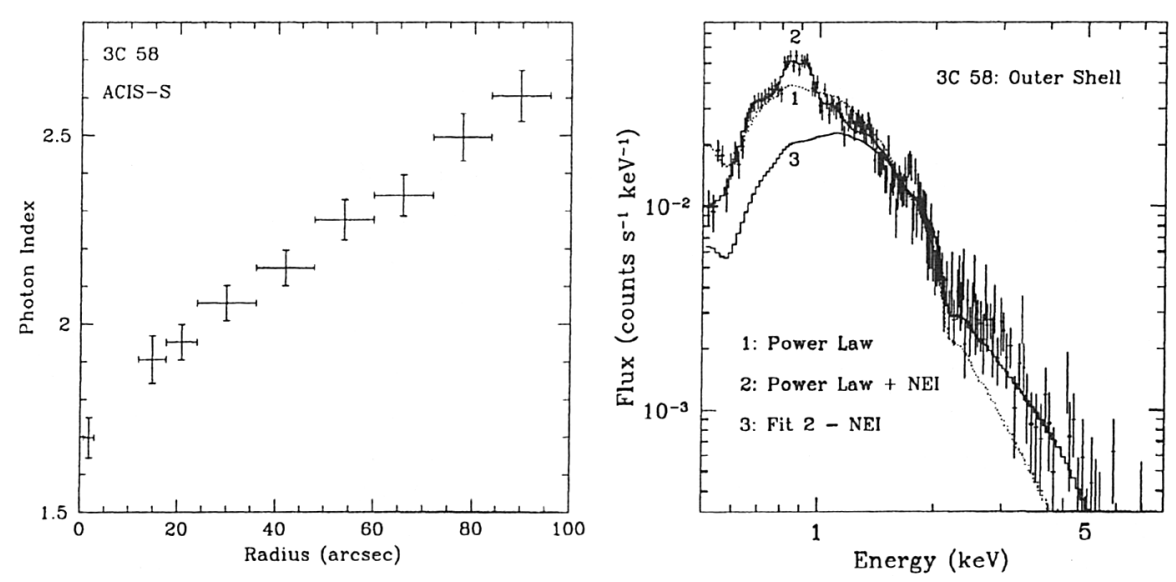

Figure 2. Left: Variation of the spectral index with radius in 3C 58. Right: Spectrum of the outermost regions showing the best-fit power law model (1); a power law + thermal model (2) that requires an overabundance of neon; and the contribution from only the power law (3) from the best-fit two component model.

\subsection{Spatial and Spectral Structure in $3 C 58$}

$\mathrm{X}$-ray spectra were extracted from various regions of $3 \mathrm{C} 58$, with corresponding background spectra taken from a region of the CCD beyond the extent of the nebula. The emission is well described by a power law model, typical of synchrotron emission. The spectral index varies throughout the PWN, however. We find, in particular, that the average spectral index increases with radius (Figure 2, left), as expected for synchrotron losses as the electrons diffuse from the center, consistent with the results based on studies of 3C 58 with ASCA (Torii et al. 2000) and XMM-Newton (Bocchino et al. 2001). Here we have forced the column density to be the same for each emission region, but have left the overall value as a free parameter in the spectral fit. The best-fit value for the column density is $N_{H}=(3.69 \pm 0.06) \times 10^{21} \mathrm{~cm}^{-2}$, and we use this value when investigating the emission from discrete regions from throughout the nebula.

For the jet-like region protruding from the pulsar, we extracted spectra from three distinct regions extending across the feature. Fixing the column density for each region at that derived above, we find a spectral index of $\sim 2.0$ with no significant evidence for variations from region to region.

As noted above, one of the remarkable features revealed by the deep ACIS observation of $3 \mathrm{C} 58$ is the presence of loop-like structures throughout the nebula, suggestive of magnetic structures in which the synchrotron emission is enhanced. We have investigated the spectra from individual loops, and compared these with the emission in the "void" regions interior to the loops to search for variations that might be expected if the synchrotron-emitting particles age as they diffuse from the loop regions. While we find spectral index differences between loops in the interior and exterior regions of the nebula, the void regions have spectra similar to those of the corresponding loops. The jet-like feature 
extending eastward from the pulsar appears to merge with a loop structure to the southeast, possibly suggesting that the former is not actually a jet. However, the spectrum of this feature is flatter than for the adjacent loop. While this does not confirm the jet notion, it does indicate that this is a distinct feature.

The X-ray spectrum from the outermost region of 3C 58 (Figure 2, right) is not well fit by a simple power law. Residual emission below $\sim 1 \mathrm{keV}$ requires an additional thermal model with $k T \sim 0.25 \mathrm{keV}$ and roughly a factor of three overabundance of neon. This is similar to results reported by Bocchino et al. (2001) based on XMM-Newton observations, and represents confirmation of the long-sought thermal shell bounding $3 \mathrm{C} 58$. The density of the thermal plasma is of order $n \sim 0.05 \mathrm{~cm}^{-2}$, corresponding to an observed mass of $\sim 0.06 M_{\odot}$, although these values are quite uncertain due to the poorly defined geometry of the thermal emission region. The presence of enhanced neon suggests that the thermal emission is associated with stellar ejecta. However, if associated with reverse-shocked ejecta, this would imply the presence of a considerable sweptup ISM component outside the PWN, which is not observed. If, instead, the emission is from ejecta swept up the the PWN itself, the size, age, and spindown power for the system imply a relatively small total ejecta mass (Chevalier 2004).

\section{Conclusions}

3C 58 is a young PWN powered by an energetic pulsar. The compact interior shows evidence of structure from the pulsar wind termination shock. Limits on thermal emission from the neutron star itself require rapid cooling mechanisms such as the direct Urca process or the presence of exotic particles in the NS interior. The overall structure of the PWN itself is dominated by a significant east-west elongation, consistent with a pulsar rotation axis in this same direction, along with complex loop-like structures that may represent magnetic structures in the nebula. Thermal emission from the outer regions of the nebula appear to correspond to ejecta-rich material that is bounding the PWN.

Acknowledgments. This work was supported by NASA Contract NAS839073 and Grant GO0-1117A.

\section{References}

Begelman, M. C., \& Li, Z.-Y. 1992, ApJ, 397, 187

Bocchino, F. et al. 2001, A\&A, 369, 1078

Chevalier, R. A. 2004, Adv. Sp. Res., 33, 456

Frail, D. A., \& Moffett, D. A. 1993, ApJ, 408, 637

Murray, S. S. et al. 2002, 568, 226

Slane, P., Helfand, D. J., \& Murray, S. S. 2002, ApJ, 571, L45

Torii, K. et al. 2000, PASJ, 52, 875

Tsuruta, S. et al. 2002, ApJ, 571, L143

van der Swaluw, E. 2003, A\&A, 404, 939

Yakovlev, D. G. et al. 2002, A\&A, 389, L24 